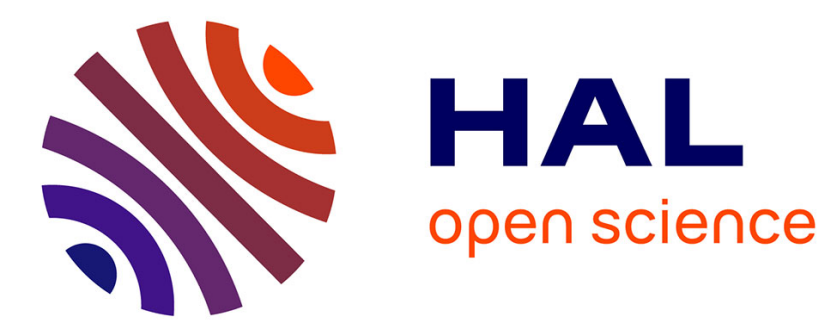

\title{
The Zero Discounting and Maximin Optimal Paths in a Simple Model of Global Warming
}

\author{
Antoine d'Autume, John M. Hartwick, Katheline Schubert
}

\section{To cite this version:}

Antoine d'Autume, John M. Hartwick, Katheline Schubert. The Zero Discounting and Maximin Optimal Paths in a Simple Model of Global Warming. Mathematical Social Sciences, 2010, 59 (2), pp.193-207. 10.1016/j.mathsocsci.2009.10.002 . halshs-00547282

\section{HAL Id: halshs-00547282 \\ https://shs.hal.science/halshs-00547282}

Submitted on 15 Dec 2010

HAL is a multi-disciplinary open access archive for the deposit and dissemination of scientific research documents, whether they are published or not. The documents may come from teaching and research institutions in France or abroad, or from public or private research centers.
L'archive ouverte pluridisciplinaire HAL, est destinée au dépôt et à la diffusion de documents scientifiques de niveau recherche, publiés ou non, émanant des établissements d'enseignement et de recherche français ou étrangers, des laboratoires publics ou privés. 


\title{
The Zero Discounting and Maximin Optimal Paths in a Simple Model of Global Warming
}

\author{
Antoine d'Autume \\ Paris School of Economics and Université Paris 1 Panthéon-Sorbonne \\ John M. Hartwick \\ Department of Economics, Queen's University \\ Katheline Schubert \\ Paris School of Economics and Université Paris 1 Panthéon-Sorbonne
}

July 2009

\begin{abstract}
Following Stollery (1998), we extend the Solow- Dasgupta-Heal model to analyze the effects of global warming The rise of temperature is caused by the use of fossil resources so that the temperature level can be linked to the remaining stock of these resources. The rise of temperature affects both productivity and utility. We characterize optimal solutions for the maximin and zero-discounting cases and present closed form solutions for the case where the production and utility functions are Cobb-Douglas, and the temperature level is an exponential function of the remaining stock of resources. We show that a greater weight of temperature in intratemporal preferences or a larger intertemporal elasticity of substitution both lead to postpone resource use.
\end{abstract}

JEL Classification: Q32 


\section{Introduction}

Global warming and its consequences for the welfare of future generations appear more and more as one of the foremost economic issues asking for proper policies. The depletion of the stock of fossil resources plays a major role in this phenomenon. A policy such as a tax on hydrocarbon-burning is necessary to control the depletion of the stock of resource and the ensuing rise of temperature. To a large extent, this amounts to a simple reallocation of carbon dioxide emissions from today to the future. Its consequences for the welfare profile of the various generations is quite important however. Capital accumulation and technical progress may mitigate the consequences for future generations of the depletion of natural resources. There is no doubt however that laisser-faire will not yield optimal results and that government intervention is required.

The design of the optimal tax response to climate change requires the choice of a social criterion to weight the welfare levels of current and future generations ${ }^{1}$. The standard one is the discounted utilitarian criterion. Social welfare is a weighted sum of the utilities of the different generations. A positive rate of discount implies that society cares less and less for generations as they become more distant in the future. An alternative is the maximin criterion. According to the principle put forward by Rawls (1972) in A Theory of justice and introduced in the natural resources and environmental economics literature by Solow (1974), society aims at maximizing the welfare of its least-advantaged member or, in our case, generation. In regular cases, this criterion ensures that each generation enjoys the same level of utility. Another alternative is the zero discounting criterion (Ramsey (1928)). This criterion rests on the ethical principle that different generations should have equal weights in social welfare. This does not preclude, however, the admission of some substitution between the welfare levels of the different generations.

We may wonder which generations are a priori disadvantaged in an economy where fossil fuels are necessary but polluting, the present and near ones or the generations in the far future. The unavoidable reduction of the stock of oil disadvantages future generations, because on the one hand extraction is an input necessary to the production process, and on the other 
hand they suffer from the temperature increase. When society discounts utilities, the process of discounting obviously adds to this disadvantage. However, even in the absence of technical progress, capital accumulation exerts a compensating influence.

As has been shown by Dasgupta and Heal (1979) or Mitra (1980) when the sole source of welfare is consumption and damages due to global warming are not taken into account, consumption and therefore welfare are doomed to decrease and tend towards zero in the long run when society discounts utilities, but may eventually be forever rising when it does not. The feasible domain is biased in favor of present generations by positive discounting, in favor of future ones by zero discounting. In any case, even if society puts equal weights on the different generations' welfare, it has to choose to what extent it is ready to substitute current welfare for future welfare.

We shall be concerned in this article with the maximin and zero discounting criteria. The second one is more general as it includes maximin as a particular case or, more precisely, as the limit case of a zero elasticity of substitution between the welfares of different generations.

Ken Stollery (1998) developed a global warming version of the Solow (1974) maximin model $^{23}$. Temperature rising harms both productivity, for instance through lower agricultural yields in countries with an already warm and dry climate, or through detrimental health effects, and welfare, for a host of reasons, among which the migration of coastal populations due to sea-level rise, and damages from more frequent storms, heat waves, or tropical diseases. Thus temperature should appear as an argument in both the production function and the current utility of a representative agent. In Stollery's model, temperature is directly related to cumulative oil extraction, and therefore to the current remaining stock of resource. This simplifying assumption avoids the explicit modeling of the accumulation of carbon dioxide in the atmosphere. It amounts to neglect the absorption of carbon dioxide by natural sinks, thus considering that carbon emissions are irreversible. Natural carbon absorption is possibly an important feature of the global warming phenomenon ${ }^{4}$, but its extent and especially its stability are controversial, so that discarding it is probably a reasonable assumption.

The effects of temperature on production and utility are externalities, not taken into account in the market decisions of private firms and households. We assume however that a Pigovian 
carbon tax internalizes these negative externalities, and thus concentrate on optimal paths of extraction, production and consumption.

Stollery showed that the Hartwick (1977) invest resource rents applies in this extended framework. In order to follow an optimal maximin path, society should invest in capital the rents obtained from the extraction of the exhaustible resource. In other words, Genuine Saving, the sum of investment in man-made capital and the real value of the destruction of the resource stock, should be zero. Equivalently, society should consume its Net National Product, that is its GDP calculated after due allowance for the destruction of its natural resources. This well-known result offers some neat theoretical foundations to the notion of a sustainable growth path. If the current generation does not want to harm the welfare of future generations, it should not consume more than its Net National Product, properly calculated.

Stollery was also able to develop a closed-form solution for the Cobb-Douglas case when temperature only affects the production function.

We generalize these results in two directions.

Building on d'Autume and Schubert (2008a), we present a more general solution method of maximin problems which also applies to the case in which temperature affects current utility negatively as well as current goods production. We then lay out the closed-form solution for the case where production and utility are Cobb-Douglas and the function linking temperature to the remaining stock of resource is exponential, as in Stollery (1998). We also show that an aggregate of capital and resource stocks is conserved along the optimal path.

We are thus able to examine how a representative consumption path changes when the parameter linking temperature to utility increases. The larger this parameter, the more gradual the temperature rise is over the long run.

For the simpler case of temperature only affecting current goods production negatively, Stollery was able to show that current investment in produced capital was unchanging, as in Solow (1974). We show that including the effect on utility leads current investment in produced capital to rise toward a bound.

In the second place we consider the more general case of a zero discounting social criterion. Following here d'Autume and Schubert (2008b), we show that a generalized Hartwick (1977) 
rule applies. In the maximin case, total investment, including the value of the depletion of the stock of resource, should be constant. In the zero discounting case, total investment should be increasing. As Ramsey (1928) first showed in a pure growth framework, the new rule is that the level of investment, evaluated in terms of utility, should depend on the distance between the current and the long run value of utility.

We again provide closed-form solutions for the specific case considered in the first part of the paper.

\section{Stollery's model}

\subsection{Discounted utility and maximin}

Let us start with a standard discounted utilitarian version of Stollery's model:

$$
\begin{aligned}
& \max \int_{0}^{\infty} e^{-\rho t} U(c(t), T(X(t))) d t \\
\dot{K}(t)= & F(K(t), x(t), T(X(t)))-c(t) \\
\dot{X}(t)= & -x(t) \\
& K_{0} \text { and } X_{0} \text { given, }
\end{aligned}
$$

with $^{5}$

$$
U(c, T)=\frac{u(c, T)^{1-1 / \sigma}}{1-1 / \sigma} .
$$

$K(t)$ and $X(t)$ are the stocks of capital and a fossil resource at time $t$. The resource, say oil, is non-renewable and its level decreases unavoidably as the result of current extraction $x(t)$. The extracted amount is used for production, together with capital and an implicit and constant quantity of labor. The production function $F(K, x, T)$ is increasing and concave in $K$ and $x$. Due to the constancy of labor, production operates with decreasing returns to scale. Production is also a decreasing function of the temperature level $T$. The production of the unique physical good is shared between investment $\dot{K}$ and consumption $c$. 
The social welfare function is the discounted sum of current utility $U(c, T)$, with a positive rate of discount $\rho$. In order to clarify the distinction between intra and intertemporal preferences we define $U(c, T)$ as an increasing function of another function $u(c, T)$ which describes the instantaneous trade-off between consumption and temperature. The positive parameter $\sigma$ is the intertemporal elasticity of substitution between utility levels. A higher $\rho$ means that society puts less weight on the utilities of future generations. A higher $\sigma$ means that society is more ready to accept substitution between the utilities of different generations. Function $u$ is increasing in $c$, decreasing in $T$ and strictly quasi-concave.

Temperature itself is an increasing function of the stock of carbon in the atmosphere and therefore, as carbon emissions are supposed to be irreversible, a decreasing function of the remaining stock of resource $X$. As this stock necessarily decreases over time, temperature will increase over time and eventually reach a maximum level when the stock of resource is exhausted. As explained above, a higher temperature has a negative effect on both production and utility.

In the maximin version of the model, the discounted sum of utilities is replaced by the minimum of current utilities over the whole horizon. The objective becomes

$$
\max \min _{t \in[0, \infty)} u(c(t), T(X(t)))
$$

As shown in d'Autume and Schubert (2008a), the maximin solution may be recovered by making the intertemporal elasticity of substitution $\sigma$ tend to zero. This expresses the absolute reluctance of society to substitute well-being in time. The rate of discount then plays no role. If society only cares about the minimum level of all utilities, the weights attached to these utilities becomes immaterial.

\subsection{Optimality conditions for the discounted utility model}

Proposition 1 Necessary conditions for an optimal discounted utility path are the following:

$$
U_{c}=\lambda
$$




$$
\begin{gathered}
\frac{\dot{\lambda}}{\lambda}=F_{K}-\rho, \\
q=F_{x}, \\
\frac{\dot{q}}{q}=F_{K}-\left(F_{T}+\frac{u_{T}}{u_{c}}\right) \frac{T^{\prime}}{q},
\end{gathered}
$$

to which we should add the technical constraints (2) and (3) giving the evolution of $K$ and $X$, and the initial conditions (4).

Proof. These conditions are easily derived from the maximization of the Hamiltonian. $\lambda$ is the co-state variable associated with capital $K$ and $q$ the absolute value of the ratio between the two co-state variables. Note that $u_{T} / u_{c}=U_{T} / U_{c}$.

$q$ can be interpreted as the price of the resource in terms of the physical good. The marginal productivity of capital $F_{K}$ is the real rate of interest $r$.

Condition (10) is the Hotelling rule. In the standard model it reduces to the fact that the real price of a non-renewable resource should grow at a rate equal to the real interest. This condition reflects the increasing scarcity of the resource and is, more deeply, an efficiency condition linking extraction and capital accumulation. In our case, the Hotelling rule must take into account the impact of current oil extraction on global warming and the associated negative externalities.

The positive expression $\left(F_{T}+\frac{u_{T}}{u_{c}}\right) T^{\prime}$ is the marginal damage measured in terms of the produced good. The price $q$ paid by producers should be the sum of a net resource price $p$, corresponding to the scarcity rent received by the owners of the resource fields, and a Pigovian carbon tax $\tau$ designed to internalize the externalities:

$$
q=p+\tau
$$

On a competitive market, the net price $p$ of the resource grows according to the standard Hotelling rule:

$$
\dot{p}=r p .
$$


From (10), (11) and (12), we deduce

$$
\dot{\tau}=r \tau-\left(F_{T}+\frac{u_{T}}{u_{c}}\right) T^{\prime}
$$

and therefore

$$
\tau(t)=\int_{t}^{\infty} e^{-\int_{t}^{s} r(u) d u}\left(F_{T}(s)+\frac{u_{T}}{u_{c}}(s)\right) T^{\prime}(X(s)) d s .
$$

At each point in time, the carbon tax should be equal to the discounted value of future marginal damages to the environment.

This Pigovian tax varies with time and may be difficult to implement. More practical, if approximate, taxes may be considered. Sinn (2008) present a recent discussion of the issue.

\subsection{Optimality conditions for the maximin model}

Various methods for solving maximin problems have been proposed, in particular by Solow (1974), Leonard and Long (1992), Cairns and Long (2006). We rather follow the simple method presented in d'Autume and Schubert (2008a), which consists in making the intertemporal elasticity of substitution tend to zero.

Proposition 2 Necessary conditions for an optimal maximin path are the following:

$$
\begin{gathered}
u(c, X)=\bar{u} \quad \text { constant }, \\
q=F_{x}, \\
\frac{\dot{q}}{q}=F_{K}-\left(F_{T}+\frac{u_{T}}{u_{c}}\right) \frac{T^{\prime}}{q} .
\end{gathered}
$$

together with the technical constraints (2) and (3) and the initial conditions (4).

Proof. From (5), we deduce $U_{c}=u^{-1 / \sigma} u_{c}$ and therefore, using (7) and (8),

$$
\frac{\dot{u}}{u}=\sigma\left(F_{K}+\frac{\dot{u}_{c}}{u_{c}}-\rho\right)
$$

which reduces to the constancy of $u$ when $\sigma$ tends to zero. 


\section{Maximin optimal paths}

\subsection{The invest resource rents rule}

A nice way to solve the maximin problem is to use the "invest resource rents" rule introduced by Hartwick (1977):

$$
G=\dot{K}+q \dot{X}=0 \quad \text { or } \quad \dot{K}=q x .
$$

Genuine saving, or net investment, $G$ is the sum of all investments realized at a given date, taking into account the disinvestment represented by the depletion of natural resources. In the present model, it is the sum of the positive investment in physical capital and the value of the extracted amount of non-renewable resources. This sum has to be zero, which means that society should compensate for the depletion of natural resources by investing an equal amount in man-made capital. In other words, society should invest in man-made capital all the rents provided by Nature through the use of non-renewable resources. This rule appears prima facie as a natural way to preserve future generations' interests.

This rule can also be linked to Net National Product or the so-called Green National Product. GDP, the Gross Domestic Product, is gross in the sense that it does not take into account the depreciation of physical capital. Much more seriously, it does not take into account either the depreciation of natural capital. National accounting goes as if the destruction of non-renewable

resources had no cost. This important observation has led to the definition of Net National Product.

In our framework, if $Y=F(K, x, T)$ describes the national product net of the depreciation of physical capital, the true Net National Product is

$$
Y^{n e t}=Y-q \dot{X}
$$

As $\dot{K}=Y-c$,

$$
G=0 \quad \Leftrightarrow \quad Y^{n e t}-c=0 .
$$

Having zero genuine saving is equivalent to consuming Net National Product. This warrants 
that current consumption does not harm the consumption possibilities of future generations.

The problem with these new accounting notions is that they should rely on the right prices, namely on prices which support an optimal growth path of the economy. Natural resources have a market price. But even if perfect competition held, this price would a priori not reflect the externalities associated with global warming. The price $q$ appearing in genuine saving should satisfy the Hotelling rule and thus take into account the effects of temperature on production and utility. This would only be the case if the government implemented the right carbon tax. The price of oil then internalizes all the relevant externalities associated with global warming. This is what we assume in the following.

The "invest resource rent" rule has clear theoretical foundations:

Proposition 3 Together with the Hotelling rule (10), the "invest resource rents rule" (14) yields a constant utility path and is therefore a necessary condition for a regular maximin optimal path.

This propostion has been proved by Hartwick (1997) for the Solow (1974) model where only consumption appeared in the utility function. A very general form, involving an arbitrary number of capital goods and implicitly allowing for externalities has been proved by Dixit, Hammond and Hoel (1980). Stollery (1998) proved that the proposition holds for his model, where temperature appears in the production and the utility functions.

A simple calculation, along the lines of previous proofs, shows that, if the Hotelling rule (10) holds,

$$
\dot{u}=0 \quad \Leftrightarrow \quad \dot{G}-r G=0
$$

where $r=F_{K}$. Thus $G=0$ implies the constancy of $u$. Dixit, Hammond and Hoel (1980) and others proved a kind of converse, namely that $G$ cannot be different from zero on an optimal maximin path.

We retain that an optimal maximin path satisfies the "invest resource rents" rule. This will allow us to find closed-form solutions. 


\subsection{Closed Form Solutions}

Stollery was able to solve the case where temperature does not appear in utility, the production function is Cobb-Douglas and the temperature function exponential:

$$
Y(t)=K(t)^{\alpha} x(t)^{\beta} T(X(t))^{-\gamma}
$$

and

$$
T(X(t))=\bar{T} e^{-\phi X(t)}
$$

$\alpha, \beta, \gamma, \phi>0, \alpha+\beta<1, \alpha>\beta$.

$\bar{T}$ is the maximal temperature reached when oil is totally exhausted.

Stollery's solution is the following:

$$
\begin{aligned}
& K(t)=K_{0}+\frac{\beta c^{*}}{1-\beta} t \\
& X(t)=\frac{\beta}{\phi \gamma} \ln \left(1+B\left(K_{0}, X_{0}\right) K(t)^{\frac{-(\alpha-\beta)}{\beta}}\right),
\end{aligned}
$$

where the sustainable level of consumption $c^{*}$ as well as the constant $B\left(K_{0}, X_{0}\right)$ are explicit function of the levels of initial stocks.

As temperature does not appear in the utility function, the maximin path is a constant consumption path, as in Solow (1974). The main features of the Solow solution are conserved. From the Cobb-Douglas production function (15), "invest resource rents" becomes $\dot{K}=q x=$ $\beta Y$, and we have $c=(1-\beta) Y$. The saving rate is constant. As consumption, production is constant and so is investment. The stock of capital increases linearly with time, in order to compensate for the unavoidable decrease of resource extraction. The stock of resource as well as the extraction rate obviously tend to zero.

We turn to the case where current temperature affects current utility negatively and, to this end, follow d'Autume and Schubert (2008a) method.

The system characterizing the optimal maximin path is composed of the Hotelling rule (10), equation (9), "invest resource rents" (14) and $u(c, T(X))=$ constant. 
The differential system is:

$$
\begin{aligned}
\dot{K} & =\beta Y \\
\dot{X} & =-Y^{1 / \beta} K^{-\alpha / \beta} T(X)^{\gamma / \beta} \\
Y & =\frac{C(u, T(X))}{1-\beta},
\end{aligned}
$$

where $C(u, T)$ is the consumption level yielding a utility level equal to $u$ when the temperature level is $T$.

We then substitute $Y$ in equation (17), eliminate time by dividing side by side (18) and (17), and separate variables to get

$$
-\left(\frac{C(u, T(X))}{1-\beta}\right)^{-\frac{1-\beta}{\beta}} T(X)^{-\frac{\gamma}{\beta}} d X=\frac{1}{\beta} K^{-\alpha / \beta} d K
$$

This integrates to

$$
\int_{X}^{X_{0}}\left(\frac{C(u, T(\xi))}{1-\beta}\right)^{-\frac{1-\beta}{\beta}} T(\xi)^{-\frac{\gamma}{\beta}} d \xi=\frac{1}{\beta} \int_{K_{0}}^{K} \kappa^{-\alpha / \beta} d \kappa
$$

This relation rests on the assumption of a Cobb-Douglas production function, which yields the simple investment rule $\dot{K}=\beta Y$, but holds for any $u(c, X)$ utility and $T(X)$ temperature functions. It could be used to derive general properties of the optimal path, depending of the properties of the utility and temperature functions ${ }^{6}$. We rather focus on the simple case considered by Stollery, where the utility function is also Cobb-Douglas:

$$
u(c, T(X))=c T(X)^{-\varepsilon} \text { or } C(u, T(X))=u T(X)^{\varepsilon}, \quad \varepsilon \geqslant 0 .
$$

Proposition 4 Assume that the production, temperature and utility function are given by (15), (16) and (21). Then the optimal maximin solution is the following: 
i) the highest sustainable level of utility is

$$
u^{*}=(1-\beta)\left(\frac{(\alpha-\beta)}{m}\right)^{\frac{\beta}{1-\beta}} T_{0}^{-\frac{\beta m}{(1-\beta) \phi}}\left(1-e^{-m X_{0}}\right)^{\frac{\beta}{1-\beta}} K_{0}^{\frac{\alpha-\beta}{1-\beta}}
$$

where

$$
m=\frac{\varepsilon(1-\beta)+\gamma}{\beta} \phi ;
$$

ii) an aggregate of the stocks of capital and resource is conserved along the optimal path:

$$
B(K, X) \stackrel{\text { def }}{=} K^{\frac{\alpha-\beta}{\beta}}\left(e^{m X}-1\right)=B\left(K_{0}, X_{0}\right) ;
$$

iii) the evolution of the capital stock is described by the following differential equation:

$$
\dot{K}=\beta \frac{u^{*} T_{0}^{\varepsilon} e^{\varepsilon \phi X_{0}}}{1-\beta}\left(1+B\left(K_{0}, X_{0}\right) K^{-\frac{\alpha-\beta}{\beta}}\right)^{-\frac{\varepsilon \beta}{\varepsilon(1-\beta)+\gamma}}
$$

where $T_{0}=T\left(X_{0}\right)=\bar{T} e^{-\phi X_{0}}$ is the initial pre-industrial temperature.

Proof. Consider equation (20) with our specification of the different functions. Simple reasoning $^{7}$ shows that the highest sustainable utility level is attained by letting $K$ tend to infinity and $X$ to zero. The resulting equation can be solved to obtain $u^{*}$. The rest of the proposition follows.

The sustainable utility level depends positively of the initial endowments of man-made and natural capital; moreover, it depends negatively on the initial temperature.

Function $B(K, X)$ defines a family of "isoquants" or iso-utility curves. We can trace out the level of $K$ required over time as $X$ tends to zero. These "isoquants" turn out to be convex to the origin and asymptotic to the axes in the $(K, X)$ plane, indicating that the model is wellbehaved. The economy will follow the iso-utility curve corresponding to the highest sustainable utility level $u^{*}$ in a downward direction, as man-made capital substitutes for oil.

It is not possible to integrate with usual functions the differential equation (24) describing the evolution of $K(t)$. It appears however that optimal investment $\dot{K}$ is no more constant as it was in the Solow (1974) model. The result however holds in the limit, as $\dot{K}$ tends to a constant 
as $T$ and $K$ tend to infinity.

The case that Stollery solved is recovered by making $\varepsilon=0$.

\subsection{Effects of a greater weight on temperature in the utility function}

Temperature in the utility function captures the direct impact of global warming on welfare. We now investigate more thorougly how the optimal paths are affected by the value of $\varepsilon$, the parameter describing the weight of temperature in the utility function.

Let us first examine the slope of the iso-utility curves defined by equation (23). This slope is

$$
\frac{d X}{d K}=-\frac{\alpha-\beta}{\beta} \frac{1-e^{-m X}}{m} \frac{1}{K} .
$$

This slope is negative and it is easy to $\operatorname{check}^{8}$ that its absolute value is a decreasing function of $\varepsilon$. At any given point in the $(K, X)$ plane, the larger $\varepsilon$, the less steep is the iso-utility curve. As this curve is the trajectory followed by the economy, this confirms that the economy reacts by saving on the resource, on $X$.

We need however to be careful when making inferences at this point. A higher level of $\varepsilon$ implies that the oil stock will be higher for any given level of capital, $K(t)$ reached by the economy. It does not tell us that the stock will be higher at any particular date under consideration. We must examine the behaviour of $\dot{X}(t)$ and $\dot{K}(t)$.

Proposition 5 An increase of $\varepsilon$

i) decreases the initial levels of production, consumption, capital accumulation and resource extraction;

ii) increases the long run levels of production, consumption, capital accumulation.

Proof. Let $g(m, X)=\left(1-e^{-m X}\right) / m$. Derivative $g_{m}(m, X)=\left[(1+m X) e^{-m X}-1\right] / m^{2}$ is negative: the numerator is equal to 0 when $X=0$ and its derivative with respect to $X$ is $-m^{2} X e^{-m X}<0$; the numerator is therefore negative for any positive $X$.

Both $\dot{K}$ and $c$ are proportional to $Y$, and $\dot{X} / \dot{K}=d X / d K$, which is negative and obviously decreases (in absolute value) through time, as $K$ increases and $X$ decreases. 
i) Let us check that an increase in $\varepsilon$ implies an initial decrease of production, $Y$. Using equation (22), initial optimal consumption is

$$
c(0)=u^{*} T_{0}^{\varepsilon}=\Psi g\left(m, X_{0}\right)^{\frac{\beta}{1-\beta}}
$$

where

$$
\Psi=(1-\beta)(\alpha-\beta)^{\frac{\beta}{1-\beta}} T_{0}^{-\frac{\gamma}{1-\beta}} K_{0}^{\frac{\alpha-\beta}{1-\beta}}
$$

does not depend on $\varepsilon$. As $g\left(m, X_{0}\right)$ decreases in $m$, which itself increases with $\varepsilon$, we find that a higher $\varepsilon$ induces the economy to have lower levels of consumption, capital accumulation and production, initially. Since $Y(0)=K_{0}{ }^{\alpha} x(0)^{\beta} T\left(X_{0}\right)^{-\gamma}$ where $x(0)$ is the only variable factor, we infer that $x(0)$ decreases $^{9}$.

ii) The long run consumption level, reached when the oil stock is asymptotically exhausted is

$$
c_{\infty}=u^{*} \bar{T}^{\varepsilon}=u^{*} T_{0}^{\varepsilon} e^{\varepsilon \phi X_{0}}=c(0) e^{\varepsilon \phi X_{0}} .
$$

It increases with $\varepsilon$ if the following inequality holds: $\frac{g_{m}\left(m, X_{0}\right)}{g\left(m, X_{0}\right)}+X_{0}>0$. We see easily that this is equivalent to $m X_{0}>1-e^{-m X_{0}}$, which is always true. As $c$ and $\dot{K}$ are fixed and independent from $\varepsilon$ proportions of $Y$, the result follows.

This proposition is rather natural. A society more concerned with global warming (one with a larger $\varepsilon$ ) chooses a slower depletion of the resource, and this implies lower production, consumption and capital accumulation "early" in the program and higher later on. The society optimally sacrifices some more goods consumption early on, but will benefit from more consumption later on and forever. The initial decrease in consumption, and oil extraction and burning, is necessary to slow down the temperature increase. Eventually, temperature will reach the same long run level, however, as it is determined by the stock of resources to burn. A greater weight of temperature in the utility function only leads to a more gradual rise of temperature. 


\section{Zero-discounting optimal paths}

We now assume zero discounting and follow the approach introduced by Ramsey (1928). The objective function becomes

$$
\max \int_{0}^{\infty}\left[U(c(t), T(X(t)))-U^{*}\right] d t
$$

where $U^{*}$ is the constant long run level of utility.

We now assume $\sigma$ to be lower than unity in (5). Then $U(c, T)$ is negative and tends to zero if $u(c, T)$ tends to plus infinity. This implies that $U^{*}$ is equal to zero. We assume ex ante that there exist feasible paths such that $u(c, T)$ tends to infinity as $t$ tends to infinity. We derive optimality conditions, characterize the potential optimal solution and check ex post that this is indeed the case for our specification of the production and utility functions.

The maximin case is obtained as a limit case for $\sigma=0$ (d'Autume and Schubert (2008a)). Society then refuses any intertemporal substitution and, in regular cases, utility remains constant over time.

\subsection{The behavior of genuine saving}

The method proposed by d'Autume and Schubert (2008b) yields what they called a "KeynesRamsey-Hartwick" rule.

Proposition 6 Consider the zero-discounting problem (27) under the technical constraints (2) and (3) and the initial conditions (4), with $\sigma<1$. On the optimal path genuine saving satisfies the following rule:

$$
G=\dot{K}+q \dot{X}=\frac{U^{*}-U(c, T(X))}{U_{c}(c, T(X))}
$$

Proof. The result follows from Bellman ${ }^{10}$. Let $V(K, X)$ be the value function of the problem. Bellman equation is:

$$
0=\max _{c, x}\left[U(c, T(X))-U^{*}\right]+V_{K}(K, X)[F(K, x, T(X))-c]-V_{X}(K, X) x
$$


First order optimality conditions are:

$$
U_{c}=V_{K}, \quad V_{K} F_{x}=V_{X}
$$

Substituting in the Bellman equation, we obtain

$$
0=U(c, T(X))-U^{*}+U_{c}(c, T(X))\left[F(K, x, T(X))-c-x F_{x}(K, x, T(X))\right]
$$

As $F(K, x, T(X))-c-x F_{x}(K, x, T(X))=\dot{K}-q x$, the result follows.

This rule appears as a generalization of both the "invest resource rents" and the KeynesRamsey rules. It describes the evolution of genuine saving. It generalizes to Stollery's model of global warming a result proved by Ramsey (1928) in his pionneering model of growth. Using variational calculus Ramsey derived a simple formula expressing investment as a function of the stock of capital, and acknowledged the help of Keynes in the interpretation of this formula.

If society has a zero discount rate but is ready to accept intertemporal substitution, genuine savings should not be zero but positive. According to the Keynes-Ramsey rule, its level depends on the distance to the stationary point. More precisely, its value expressed in terms of utility $U_{c}[\dot{K}+q \dot{X}]$ is equal to the distance $U^{*}-U(c, T(X))$ between current utility and its long run value. Thus, the farther the economy from the stationary point, the higher its genuine savings expressed in terms of utility.

With our specification (21) of the utility function, we have

$$
\frac{U_{c}}{U(c, X)}=\left(1-\frac{1}{\sigma}\right) \frac{u_{c}}{u(c, X)}=\left(1-\frac{1}{\sigma}\right) \frac{1}{c}
$$

Taking into account $U^{*}=0$, equation (28) becomes

$$
G=\dot{K}+q \dot{X}=\frac{\sigma}{1-\sigma} c
$$

With our Cobb-Douglas production function, $q \dot{X}=-\beta Y$ so that $\dot{K}=\beta Y+\frac{\sigma}{1-\sigma} c$ which, together with $\dot{K}=Y-c$, yields the rule of production sharing between consumption and 
investment along the optimal path, and the value of genuine savings:

$$
\begin{aligned}
c & =(1-\widehat{\beta}) Y, \\
\dot{K} & =\widehat{\beta} Y \\
G & =(\widehat{\beta}-\beta) Y
\end{aligned}
$$

with

$$
1-\widehat{\beta}=(1-\beta)(1-\sigma)
$$

The saving rate $\widehat{\beta}$ lies between $\beta$ and 1 , and is equal to $\beta$ when $\sigma=0$. Consumption, investment and genuine savings all are constant shares of the gross national product. In the maximin case,

$\sigma=0$ and $\widehat{\beta}=\beta$ so that genuine savings is equal to zero. When $\sigma>0$, genuine savings is positive and even growing, as we shall check that $Y$ is increasing. This of course does not contradict the Keynes-Ramsey-Hartwick rule as marginal utility $U_{c}$ decreases so that genuine savings expressed in terms of utility decreases to zero.

\subsection{Closed-form solution}

The envelope theorem allows us to obtain the evolution of the shadow prices. Let

$$
\lambda=U_{c}(c, T(X))=V_{K}, \quad \mu=V_{X}
$$

We get

$$
\begin{gathered}
\frac{\dot{\lambda}}{\lambda}=-F_{K}, \\
\frac{\dot{\mu}}{\mu}=-\left(F_{T}+\frac{u_{T}}{u_{c}}\right) \frac{T^{\prime}}{q} .
\end{gathered}
$$

The price of the resource stock in terms of capital is $q=\mu / \lambda$ so that

$$
\frac{\dot{q}}{q}=F_{K}-\left(F_{T}+\frac{u_{T}}{u_{c}}\right) \frac{T^{\prime}}{q} .
$$


The Hotelling rule is the same as in the discounting or maximin case (equation (10)). This is not surprising as this rule is a pure efficiency condition, which does not depend on the choice of an intertemporal criterion.

The use of the Cobb-Douglas utility function (21) yields an explicit characterization of the behavior of the shadow prices and therefore of the marginal values of both stocks.

Proposition 7 i) On the optimal path, the value of capital can be expressed as a function of the sole capital stock, while the value of the resource can be expressed as a function of the sole resource stock:

$$
W_{K}=\lambda K=B_{1} K^{1-\frac{\alpha}{\hat{\beta}}}, \quad W_{X}=\mu X=B_{2} X T(X)^{-\frac{\widehat{m}}{\phi}},
$$

where $B_{1}$ and $B_{2}$ are constants depending on initial conditions, and

$$
\widehat{m}=\frac{\varepsilon(1-\widehat{\beta})+\gamma}{\beta} \phi
$$

ii) An optimal path exists if and only if

$$
\alpha>\widehat{\beta}
$$

that is, if and only if

$$
\alpha>\sigma+\beta(1-\sigma)
$$

iii) On the optimal path, $K$ tends to infinity while $X$ tends to zero.

Proof. i) With Cobb-Douglas production and utility functions, equations (29) and (30) take the form

$$
\begin{gathered}
\frac{\dot{\lambda}}{\lambda}=-\alpha \frac{Y}{K}=-\frac{\alpha}{\widehat{\beta}} \frac{\dot{K}}{K}, \\
\frac{\dot{\mu}}{\mu}=\frac{\gamma Y+\varepsilon c}{\beta \frac{Y}{x}} \frac{T^{\prime}(X)}{T(X)}=-\frac{\widehat{m}}{\phi} \frac{\dot{T}}{T},
\end{gathered}
$$

Thus the shadow price of capital can be expressed as a function of the sole capital stock, while 
the shadow price of the resource can be expressed as a function of the sole temperature:

$$
\lambda=B_{1} K^{-\frac{\alpha}{\hat{\beta}}}, \quad \mu=B_{2} T(X)^{-\frac{\widehat{m}}{\phi}},
$$

where $B_{1}$ and $B_{2}$ are constants. The values $W_{K}$ et $W_{X}$ of the capital and the resource stocks follow.

ii) and iii) $W_{K}$ tends to zero as time tends to infinity iff $\alpha<\widehat{\beta}$ and $K$ tends to zero, or $\alpha>\widehat{\beta}$ and $K$ tends to infinity. If the capital stock were to tend to zero, production and consumption would do the same as the resource input also has to tend to zero. This cannot be optimal in a model with zero discounting. (31) follows. $W_{X}$ tends to zero as time tends to infinity if and only if $X T(X)^{-\frac{\widehat{m}}{\phi}}$ tends to zero. As $T(X)$ must be finite, $X$ has to tend to zero.

Condition (31) is more stringent that the condition $\alpha>\beta$ required in the maximin case. Moreover, it involves technological parameters only in the maximin case, whereras it involves here both technological and preference parameters. Along the optimal path, the capital stock grows without limit, in order to maintain an increasing consumption in spite of the decrease in resource use. The resource is asymptotically exhausted.

Proposition 8 The optimal solution for an optimal maximin path is the following:

i) An aggregate of the stocks of capital and resource is conserved along the optimal path:

$$
\widehat{B}(K, X) \stackrel{\text { def }}{=} K^{\frac{\alpha-\widehat{\beta}}{\widehat{\beta}}}\left(e^{\widehat{m} X}-1\right)=\widehat{B}\left(K_{0}, X_{0}\right)
$$

ii) The evolution of the capital stock is described by the following differential equation:

$$
\dot{K}=\hat{\beta} \widehat{A}\left[1+\widehat{B}\left(K_{0}, X_{0}\right) K^{1-\frac{\alpha}{\hat{\beta}}}\right]^{-\frac{\varepsilon(1-\widehat{\beta})}{\varepsilon(1-\widehat{\beta})+\gamma} \frac{\beta}{1-\beta}} K^{\frac{\alpha(\widehat{\beta}-\beta)}{\beta(1-\beta)}}
$$

iii) The instantaneous utility level

$$
u=(1-\widehat{\beta}) \bar{T}^{-\varepsilon} \widehat{A}\left[1+\widehat{B}\left(K_{0}, X_{0}\right) K^{1-\frac{\alpha}{\beta}}\right]^{\frac{\varepsilon \beta}{\varepsilon(1-\widehat{\beta})+\gamma}\left(1-\frac{1-\widehat{\beta}}{1-\beta}\right)} K^{\frac{\alpha(\widehat{\beta}-\beta)}{\widehat{\beta}(1-\beta)}}
$$


is an increasing function of $K$ and therefore increases without limit along the optimal path. This implies that $U=u^{1-1 / \sigma} /(1-1 / \sigma)$ tends to $U^{*}=0$ as time and capital tend to infinity.

Proof. i) The Hotelling rule (10) now reads

$$
\frac{\dot{q}}{q}=\frac{\alpha}{\hat{\beta}} \frac{\dot{K}}{K}-\frac{\widehat{m}}{\phi} \frac{\dot{T}}{T}
$$

This shows that $q$ can be expressed as a simple function of $K$ and $T(X)$ :

$$
q=\Phi_{0} K^{\frac{\alpha}{\hat{\beta}}} T(X)^{-\frac{\widehat{m}}{\phi}}
$$

with

$$
\Phi_{0}=q_{0} K_{0}^{-\frac{\alpha}{\hat{\beta}}} T\left(X_{0}\right)^{\frac{\widehat{m}}{\phi}}
$$

As in the maximin case, the solution is obtained by time elimination and variable separation.

The ratio of the two equations of motion $\dot{K}=\widehat{\beta} Y$ and $\dot{X}=-x=-\beta \frac{Y}{q}$ yields, using (35):

$$
\frac{d X}{d K}=-\frac{\beta}{\widehat{\beta}} \frac{1}{q}=-\frac{1}{\Phi_{0}} \frac{\beta}{\widehat{\beta}} K^{-\frac{\alpha}{\hat{\beta}}} T(X)^{\frac{\widehat{m}}{\phi}} .
$$

Thus we obtain

$$
T(X)^{-\frac{\widehat{m}}{\phi}} d X=-\frac{1}{\Phi_{0}} \frac{\beta}{\widehat{\beta}} K^{-\frac{\alpha}{\hat{\beta}}} d K
$$

which integrates in

$$
\int_{X_{0}}^{X} T(\xi)^{-\frac{\widehat{m}}{\phi}} d \xi=\frac{1}{\Phi_{0}} \frac{\beta}{\alpha-\widehat{\beta}}\left(K^{1-\frac{\alpha}{\widehat{\beta}}}-K_{0}^{1-\frac{\alpha}{\hat{\beta}}}\right) .
$$

In order to get an explicit solution we revert to Stollery's temperature function (16) so that the previous equation becomes

$$
\frac{1}{\widehat{m}}\left(e^{\widehat{m} X}-e^{\widehat{m} X_{0}}\right)=\frac{1}{\Phi_{0}} \frac{\beta}{\alpha-\widehat{\beta}}\left(K^{1-\frac{\alpha}{\widehat{\beta}}}-K_{0}^{1-\frac{\alpha}{\hat{\beta}}}\right) .
$$


Making $X \rightarrow 0$ and $K \rightarrow \infty$ in equation (37) then yields, as $\alpha>\widehat{\beta}$,

$$
\frac{1}{\widehat{m}}\left(1-e^{\widehat{m} X_{0}}\right)=-\frac{1}{\Phi_{0}} \frac{\beta}{\alpha-\widehat{\beta}} K_{0}^{1-\frac{\alpha}{\hat{\beta}}}
$$

which determines $\Phi_{0}$ and therefore $q_{0}$, by equation (36):

$$
q_{0}=\Phi_{0} K_{0}^{\frac{\alpha}{\hat{\beta}}} e^{\widehat{m} X_{0}}=\frac{\beta \widehat{m}}{\alpha-\widehat{\beta}} \frac{K_{0}}{1-e^{-\widehat{m} X_{0}}}
$$

We also have, from (37),

$$
\frac{1}{\widehat{m}}\left(e^{\widehat{m} X}-1\right)=\frac{1}{\Phi_{0}} \frac{\beta}{\alpha-\widehat{\beta}} K^{1-\frac{\alpha}{\widehat{\beta}}}
$$

Dividing side by side equations (40) and (38) allows us to obtain (32).

ii) From (35), (38) and (32) we obtain

$$
\begin{aligned}
\frac{q}{K} & =\Phi_{0} K^{\frac{\alpha}{\hat{\beta}}-1} e^{\widehat{m} X}=\frac{\beta \widehat{m}}{\alpha-\widehat{\beta}} \frac{K_{0}^{1-\frac{\alpha}{\hat{\beta}}}}{e^{\widehat{m} X_{0}}-1} K^{\frac{\alpha}{\hat{\beta}}-1} e^{\widehat{m} X} \\
& =\frac{\beta \widehat{m}}{\alpha-\widehat{\beta}} \frac{1}{e^{\widehat{m} X_{0}}-1}\left(\frac{K_{0}}{K}\right)^{1-\frac{\alpha}{\hat{\beta}}}\left[1+\left(\frac{K_{0}}{K}\right)^{\frac{\alpha}{\hat{\beta}}-1}\left(e^{\widehat{m} X_{0}}-1\right)\right]
\end{aligned}
$$

Using $q=\beta Y / x$ with $x$ taken from the production function (15) and using (32) again yields

$$
\frac{\widehat{m}}{\alpha-\widehat{\beta}} \frac{1}{e^{\widehat{m} X_{0}}-1}\left(\frac{K_{0}}{K}\right)^{1-\frac{\alpha}{\hat{\beta}}}\left[1+\left(\frac{K_{0}}{K}\right)^{\frac{\alpha}{\hat{\beta}}-1}\left(e^{\widehat{m} X_{0}}-1\right)\right]^{1-\frac{\gamma \phi}{\beta \hat{m}}}=Y^{1-\frac{1}{\beta}} K^{\frac{\alpha}{\beta}-1} \bar{T}^{-\frac{\gamma}{\beta}}
$$

Then

$$
Y=\widehat{A}\left[1+\widehat{B}\left(K_{0}, X_{0}\right) K^{1-\frac{\alpha}{\widehat{\beta}}}\right]^{-\frac{\varepsilon(1-\widehat{\beta})}{\varepsilon(1-\widehat{\beta})+\gamma} \frac{\beta}{1-\beta}} K^{\frac{\alpha(\widehat{\beta}-\beta)}{\hat{\beta}(1-\beta)}}
$$

where $\widehat{A}=\left(\frac{(\alpha-\widehat{\beta}) \widehat{B}}{\widehat{m}}\right)^{\frac{\beta}{1-\beta}} \bar{T}^{-\frac{\gamma}{1-\beta}}$ is a positive constant. This equation yields, using $\dot{K}=\widehat{\beta} Y$, the differential equation in $K(33)$, impossible to integrate with the usual functions. It is easy to check that this equations reduces to (24) when $\sigma=0$. 
iii) We now turn to the expression of $u$. We have

$$
u=c T(X)^{-\varepsilon}=(1-\widehat{\beta}) Y \bar{T}^{-\varepsilon} e^{\varepsilon \phi X},
$$

which, using equations (41) and (32), yields equation (34). As $\alpha>\widehat{\beta}>\beta, u$ is an increasing function of $K$ and therefore increases without limit along the optimal path.

Equation (32) defines a family of trajectories in the $(K, X)$ plane. Initial endowments $\left(K_{0}, X_{0}\right)$ determine the relevant trajectory. The economy follows this curve in a downward direction, as man-made capital substitutes for natural capital. The capital stock tends to infinity, as the resource stock tends to zero. In the maximin case, these trajectories, defined by equation (23), are iso-utility curves. When $\sigma \neq 0$ utility is growing along each one of these trajectories.

\section{Concluding Remarks}

Thus Stollery's global warming model represents an interesting and elegant extension of Solow (1974). Increased consumption compensates agents for the disutility implied by the unavoidable rise in temperature and allows them to maintain a constant level of utility. We have been able to complement Stollery's analysis and to provide a closed-form solution for the case where temperature also has a direct effect on utility. This allowed us to identify the role played by a temperature externality in utility. It leads to less physical production and consumption in the short run, in order to save the resource and prevent an excessive rise in temperature. But it also boosts consumption and production later on and forever, as capital accumulation benefits from the postponement of the rise in temperature.

We also considered a more general zero-discounting criterion which enables us to examine the effects of greater acceptance of intergenerational substitution of welfare levels, and show that it plays to the advantage of future generations. To the contrary, a maximin criterion appears to protect current generations.

Our results are dependent on the two assumptions of Cobb-Douglas production and utility. The first one captures in a simple way the essential role of the resource as it states that 
it is impossible to produce without resource. This does not prevent the resource stock from converging to zero, but only at the limit when time goes to infinity and an infinite amount of produced capital, in the limit of time, become available to substitute for the vanishing resource. An interesting extension to consider is the case of a CES utility function, with limited substitution between consumption and the temperature disamenity. As shown in a slightly different context in d'Autume and Schubert (2008a), the optimal solution would then be not only to postpone extraction but also to maintain forever some finite portion of the oil stock, an amount endogenously determined, thus preventing the temperature from reaching its maximal level.

\section{Notes}

\footnotetext{
${ }^{1}$ We do not study intra-generational inequality and are only concerned with the welfare of a representative member of each generation.
}

${ }^{2}$ We honor the memory of researcher Ken Stollery (1948-2005) who was struck down by illness at a tragically early age.

${ }^{3}$ Hamilton and Ulph [1995] developed a somewhat different version of a Solow model with global warming independently of Stollery.

${ }^{4}$ See for instance Farzin and Tahvonen (1996).

${ }^{5}$ We drop the time argument when no ambiguity arises.

${ }^{6}$ D'Autume and Schubert(2008a) show that a CES utility function implies that society will choose to conserve forever a strictly positive level of resource, which is endogenously determined.

${ }^{7}$ See d'Autume and Schubert (2008a).

${ }^{8}$ See the proof of the following proposition.

${ }^{9}$ This result also derives from the fact that $x(0) / \dot{K}(0)=-d X(0 / d K(0)$ decreases with $\varepsilon$.

${ }^{10}$ It also derives from the nullity of the Hamiltonian.

\section{References}

[1] D'Autume, Antoine and Katheline Schubert, 2008a, "Hartwick's Rule and Maximin Paths when the Exhaustible Resource has an Amenity Value", Journal of Environmental Eco- 
nomics and Management, 56, 3, 260-274.

[2] D'Autume, Antoine and Katheline Schubert, 2008b, "Zero Discounting and Optimal Paths of Depletion of an Exhaustible Resource with an Amenity Value", Revue d'Economie Politique, 119, 6, 827-845.

[3] Dasgupta, Partha, and Geoffrey Heal, 1979, Economic theory and exhaustible resources, Cambridge University Press.

[4] Dixit, Avinash K., Peter Hammond, and Michael Hoel, 1980, "On Hartwick's Rule for Regular Maximin Paths of Capital Accumulation and Resource Depletion", Review of Economic Studies, 47, 3, 551-56.

[5] Farzin, Y. Hossein and Olli Tahvonen, 1996, "Global Carbon Cycle and the Optimal Time Path of a Carbon Tax", Oxford Economic Papers, 48, 4, 515-536.

[6] Hamilton, Kirk and David Ulph, 1995, "The Hartwick Rule in a Greenhouse World", unpublished manuscript, University College, London.

[7] Hartwick, John, 1977, "Intergenerational Equity and the Investing of Rents from Exhaustible Resources", American Economic Review, 66, 5, 972-974.

[8] Leonard, Daniel and Ngo Van Long, 1992, Optimal Control Theory and Static Optimization in Economics, New York: Cambridge University Press.

[9] Mitra, Tapan, 1980, "On Optimal Depletion of Exhaustible Resources: Existence and Characterization Results", Econometrica, 48, 6, 1431-1450.

[10] Ramsey, Frank, 1928, "A Mathematical Theory of Savings", Economic Journal, 38, 543559.

[11] Rawls, John, 1972, A Theory of Justice, Clarendon Press, Oxford.

[12] Sinn, Hans-Werner, 2008, "Public policies against global warming: a supply side approach", International Tax and Public Finance, 15, 360-394. 
[13] Solow, Robert M., 1974, "Intergenerational Equity and Exhaustible Resources", Review of Economics Studies, Symposium volume, 29-46.

[14] Stollery, Kenneth R., 1998, "Constant Utility Paths and Irreversible Global Warming", Canadian Journal of Economics, 31, 3, 730-42. 